\title{
The Strategy for English Website Construction and Its Significance in Teaching and Guidance
}

\author{
Yuanyuan Song \\ Xi'an University, School of Foreign Studies, 710065
}

\begin{abstract}
Keywords: English website; Construction strategy; Teaching; Guidance; Significance
\end{abstract}
\begin{abstract}
The construction of English website firstly needs a strict planning; the development and design of the English website should be on the basis of the definite nature and the theme positioning of the website, the existing research results, and the combination with mode of thinking and reading habits of foreign audiences. English website is bound to reflect a certain content and purpose of design. In order to make the English website in line with foreign style and cultural habits, it is necessary to construct the website by using the Internet to search information as well as considering the psychology of the audience. We cannot put all the foreign knowledge or post the Chinese contents remaining unchanged on the website; we should achieve a combination of the two while constructing the website. It is hoped to better summarize the English website construction strategy through studying how to combine with the enterprise and according to a certain teaching method in the process of teaching the English website construction strategy.
\end{abstract}

\section{Introduction}

English website construction requires the optimization of the search engine. The criterion for the optimization is that whether the needed content can be quickly searched by foreigners. In order to achieve SEO optimization, it is needed to submit the own website on the Internet, and achieve better promotion at home and abroad in the process of indexing the site. The marketing and promotion of English website need to enhance the browsing and recommendation in the foreign information groups. In order to publicize the English website, the developer of the website can try to release a variety of posts and advertorials on the forums while browsing the well-known websites and forums at home and abroad, and do a good job in dealing with the creative ideas and details of the English website in the process of construction of the English website. It is necessary to educate the talents in universities and colleges, training high-quality talents for English website construction and planning.

\section{Research Background of Modern Enterprise English Website Construction}

The Rapid Development of the Internet Brings Challenges for the Enterprise/ The rapid development of the Internet has brought new challenges to the enterprise. The development of the Internet has exponentially increased the number of Internet users at home and abroad; especially, the Internet users aged between 18 and 50 years account for about $80 \%$ of the total number of Internet users. The quality of Internet users continues to improve; therefore, the browsing requirements for the English site become higher. At present, in order to faster obtain network information and resources, under the influence of the huge number of Internet users and convenient shopping environment and browsing environment, the development of the Internet breaks the restrictions on the website operation of the enterprise, and there is no obvious regional distinction between the enterprises. The exchange of goods and information can be continuously involved in the process of internationalization and global competition; the transmitting costs and transaction costs between enterprises are constantly reduced, even small and medium enterprises can also rejuvenate in the new era of the Internet. Therefore, the Internet as an information platform brings a huge opportunity to the enterprises. The English website as an important link for the communication between enterprises and the world is valued. The high-level professional planning talent is urgently needed in the process of enterprise development. 
The Development of College Language Teaching and the Background of Enterprise English Website Construction. The latest survey on website development shows that the language translation education in colleges and universities has now stepped into a developmental stage with differentiation, and the English website construction with rapid development, compared with the Chinese websites with maturity and steady development, still has a certain shortcoming in website configuration. English language development and website construction are relatively disjointed, and the construction of English website has become a problem to be solved for a lot of enterprises. The current efficient English website construction provides reference for the future construction of English website. The Internet has been developed in China for decades, and the development of the site construction is very rapid, and there are also a lot of requirements for the enterprise English website. The colleges and universities, with regard to the education awareness and skills development in construction of English website, believe that the English website should be able to serve for the enterprise and also provide reference for information exchange. However, in the future, whether the English site construction and planning talents are able to work as a professional team highly depends on that whether the team can meet the new needs of the market economy. The importance of the English website is self-evident. Schools and enterprises need to achieve the consensus in the English website construction. Through the researches on the English site construction of universities of "985 Project" or other colleges and universities, the data can be realtime updated. The investigation and analysis of planning and construction of English website can provide a lot of important data. The construction of news websites is not only to simply translate the contents of Chinese website, but also to provide channels and platforms to enhance international exchanges and achieve interoperability between English and Chinese, to achieve exchanges of cultures and information.

\section{The Present Situation of College English Website Translation and Practical Training and Teaching in Enterprises}

The Translation on English Web Pages of Colleges and Universities is not Standardized. At present, the English translation on the English websites of many colleges and universities is not standardized. Many English sites are directly translated based on the Chinglish, which is a simple translation of the Chinese web page, and even there will be typo and ungrammatical sentences, which is very unprofessional. In fact, with the development of the Internet, downloading information is actually very convenient, but the English expression on college English web pages after translation is not accurate, or can not use the information on western advanced English website for reference, which is very troublesome. Parallel text cannot provide professional English expression, or even cannot guarantee the accuracy. In the translation process, the translation version is relatively inadequate, and the settings of webpage items are complex, so translation of the contents of the original Chinese websites and retaining of all the original items make the website revision cannot be achieved effectively. The English website construction cannot use the advanced western experience for reference to achieve academic exchanges and the introduction of advanced culture, making the English site become useless.

The Chinglish is Common on English websites of Enterprises. The Chinglish is common on the English websites of enterprises. The Chinglish only retains the structure of original text, breaking the principles and sense of beauty of English translation and reducing the qualified vocabulary expression, thus even a very short introduction of the enterprises or product introduction cannot be effectively expressed in English. In the process of constructing English website, the reflection and summary for translation practice is not enough, and it is required to complete a lot of work within a short period of time. As a result, the English expression of the entire website is not idiomatic enough, thus the English website visitors will glance the content or just ignore it while browsing the website.

English Website Constructor-based Thought is Prevalent. The website construction is based on the thought of constructor, instead of the thought of website visitors. The neglect of the purpose of site construction makes the website construction superficial. Inadequate preparation before translation and 
limited reference for translation make the English site translator cannot correctly express the original contents in English, thus the English website visitors cannot better understand the contents of the website.

The Universality and Timeliness of Enterprise English Website is Poor. The English website of the enterprise should be a platform to reflect the time-based change of English knowledge and the change of the advanced English information in the world. At present, the effectiveness of construction of the English website in our country is very poor, because the relevant professional translation talents are limited, and the news collecting and editing staff cannot meet the needs of the position. Therefore, in the process of enterprise English website construction, the timeliness of each update and revision is very poor. If the website visitors cannot achieve the purpose of utilizing the English website, and the site constructor cannot realize the best universality and timeliness of the English website, the English site may be eliminated after a period of time.

\section{The Combination of Actual English Website Construction and the Teaching in Colleges and Universities}

Enhance the Whole Society and Enterprises' Awareness on Importance of English Website Construction. It is vital to enhance the awareness of the whole society and the colleges and universities on the importance of the English websites construction. As a link for global communication, the English website is constructed to understand the interactive needs within the whole society. We should focus on the key procedures of website construction and follow the procedures of combination of centralization and decentralization to enhance the translation and framework of the website.

Persist the Original Intention of Displaying and Promoting the International Image of Brand. English website should serve for the enterprises as well as for the site users and promoters. Therefore, the English website not only serves for foreign brands, but also for the Chinese brands. The English website plays a role in displaying and promoting the brand, creating a new international image for the enterprise, which is the purpose and the original intention of enterprise development. Therefore, the enterprises should promote their international brands with different brand images and brand advertising on the website, to ensure the success of English website construction.

Strengthen the Link between Colleges and Universities Teaching and Social Enterprises at Home and Abroad. The training base of colleges and universities and enterprises is basic to ensure the ultimate purpose of teaching of English website construction. Strengthening the combination of domestic and foreign education experience in colleges and universities, enhancing the close contact between social enterprises and colleges and universities, and understanding the causal relationship between the actual effects of education in colleges and universities and social enterprises can improve the level of talents for enterprise management and website construction within the whole society. Through the training, social organizations and enterprises can make up for the weaknesses in the strategy and arrangements while understanding the construction of the English website, thus can effectively construct the English websites of colleges and universities. It should be noted that the connection between enterprises and institutions and colleges and universities should be enhanced. We should take the English website construction as the actual inquiry-based learning project, students as the main body of learning, and refine and upgrade the theory of English website construction in practice, as well as enhance and speed up the construction of the English site, so as to make the English website construction and planning talent more adaptable to the needs of future development of enterprises, being able to advance with the times to understand the needs of modern English website construction.

Improve the Efficiency of Enrollment of Foreign Students and Recruitment of Experts. Website construction requires the assistance of professional talents. Therefore, it is needed to strengthen the enrollment of foreign students and improve the frequency of recruitment of foreign experts for construction of the English website. In the process of website construction, it is recommended for the enterprises, especially small and medium enterprises, to use specialized and 
experienced experts to construct the website. The faculty of colleges and universities should also be adjusted accordingly, because the construction of the site needs not only professional planning talent, but also the foreign teachers to give professional courses for website construction. Therefore, enrollment of foreign students and recruitment of foreign experts and teachers are complementary; especially, the foreign students can understand the culture of China and their own countries or multi-national culture. Therefore, the website construction and exchanges of culture can be done well.

Make the Information Dissemination and Translation Architecture More Comprehensive. The English website is constructed to carry out brand promotion, sales and marketing strategy, as well as comprehensive and reasonable planning of information dissemination and translation architecture. Therefore, in order to make the information dissemination and translation architecture of English website more comprehensive, we must use different media for promotion. With regard to the information dissemination and translation architecture, through the improvement of skills in translating the information we can ensure that English websites of colleges and universities and enterprise can be constructed effectively and perfectly, enhancing the rationality of English website, as well as the unified planning of the details of each item and rational allocation of various parts of the site.

\section{Construct the English Website from the Perspective of Audience}

It is required to handle the creative design and details of website properly, strengthen the web design style of English website, and consider from the perspective of the English site audience. The style of English website should be entirely different from that of the domestic website which emphasizes the gaudiness. The English website should be concise in style, emphasizing the details of the site design to meet the preferences of foreign audiences, and the website should choose a good international domain name. Developing and constructing the website from the perspective of the English site visitors can make the English website become more competitive in the international market. In order to make the website operation not limited to the domestic construction style, it is necessary to build the own brand characteristics of the English website. To maintain the activeness of website, we can release a variety of promotional advertorials related to the website, pay attention to the frequency of website updates and language accuracy, and achieve interaction between website visitors. We should also pay special attention to the English language habits to enhance the quality of the content and the update frequency, so as to better promote the brand.

\section{Conclusion}

At present, from the perspective of educating and training talent for English website, it was found in the study that the structure of the enterprise website is single compared to that of the website of colleges and universities. If the enterprise website want to achieve standardization and professional standards, it needs to achieve the overall optimization and design of website contents under a variety of website menus. It is necessary to strengthen the education of English website construction in colleges and universities, enhance the informationization and internationalization level of English website, so as to enable the construction of English websites with different natures to reach higher levels. Website reflects the essence of enterprise management and operation. We can reduce the actual differences between Chinese and English website from the aspect of mode and product form, ensuring better planning of the enterprise English website from the perspective of accurate expression.

\section{Acknowledgements:}

Research on the problems and strategies of English websites in enterprises under the background of export-oriented development . 


\section{References}

[1] Li X.F., Wang C. Analysis on the Current Situation of Construction and Management of English Portal Website of Xi'an Municipal Government and Its Promotion Strategy [J]. Automation \& Instrumentation,2017,(08):96-98. [2017-10-05]. DOI: 10.14016/j.cnki.10019227.2017.08.096

[2] Qiu Z.H., Luo Y.Y., Qin P.Y. An Analysis of the Construction of English Websites in the Times of Internet [J]. Guangxi Education,2017,(23):50-51+88. [2017-10-05].

[3] Yan X.H. A Contrastive Study of Cross-cultural Communication between Chinese and Foreign Universities Website [J/OL]. New Media Research,2016,2(23):115-117+138. (2016-1215)[2017-10-05]. http://kns.cnki.net/kcms/detail/10.1330.G2.20161215.1720.114.htmIDOI : 10.16604/j.cnki.issn2096-0360.2016.23.057

[4] Chen J. An Investigation and Analysis of the Current Situation of English Course of NonEnglish Majors in Colleges and Universitie [D]. Shandong Normal University,2016.

[5] Wang Q. Research on Compiling Strategies in Translation of Chinese Universities Website Introduction [D]. Jiangsu University of Science And Technology,2015.

[6] Xu D.E. A Study on the Optimization Strategy of Local Government English Website [J]. Media,2015,(03):79-80. [2017-10-05].

[7] Yang Q. An Analysis of the Construction of English Websites of Colleges and Universities in Guizhou Province under the Evaluation Index System [J]. Journal of Hubei University of Education,2014,31(07):90-92. [2017-10-05].

[8] Pei L., Zhou L.Q. Construction of Chinese University English Website Group from the Perspective of Internationalization of Higher Education - A Comparative Study with 20 Worldrenowned English Websites [J]. Contemporary Education and Culture,2014,6(03):61-66. [2017-10-05]. DOI: 10.13749/j.cnki.cn62-1202/g4.2014.03.011

[9] Dai J.C. Translation Problems and Countermeasures of Foreign Publicity Website from the Perspective of Skopos Theory: A Case Study of Some English Websites in Wenzhou [J]. Journal of Changchun University of Science and Technology,2014,27(03):123-126. [2017-1005].

[10]Luo M. An Analysis of the Current Situation of Local Government 's English Website Construction and the Strategy of Promotion [J]. Success,2013,(24):292-293. [2017-10-05].

[11]Luan J., Liu W. Current Situation and Strategy of English Portal Website Construction in Sports Colleges and Universities in China [J]. Journal of Shanghai University of Sport,2012,36(01):47-50. [2017-10-05]. DOI: 10.16099/j.cnki.jsus.2012.01.003

[12] Shi Z.Z. Research on Information Construction of English Learning Resources Website [D]. Tianjin Foreign Studies University,2013. 